\title{
PRODUCCIÓN Y CALIDAD DE LA BIOMASA DE MORERA (Morus alba) FERTILIZADA CON DIFERENTES ABONOS ${ }^{1}$
}

\author{
Jorge Alberto Elizondo-Salazar ${ }^{2}$
}

\begin{abstract}
RESUMEN
Producción y calidad de la biomasa de morera ( $\mathrm{Mo}$ rus alba) fertilizada con diferentes abonos. Se llevó a cabo un experimento en la Estación Experimental "Alfredo Volio Mata" de la Universidad de Costa Rica con el fin de evaluar la aplicación de $150 \mathrm{~kg}$ de N/ha/año proveniente de dos abonos orgánicos: lombriabono y compostaje; y de un fertilizante químico, sobre la producción y calidad de la biomasa de morera. El periodo experimental comprendió un ciclo de 12 meses, iniciando en julio del 2003 y finalizando en julio del 2004. Se utilizó una plantación de morera de 12 años de establecida con una densidad de siembra de 27.777 plantas/ ha. Se empleó un diseño de bloques completos al azar con cuatro tratamientos: dos abonos orgánicos, nitrato de amonio $(33,5 \% \mathrm{~N})$ y un control. Las plantas se podaron a $0,6 \mathrm{~m}$ sobre el nivel del suelo al inicio del ensayo. Durante el periodo experimental, las plantas fueron podadas consecutivamente cada 90 días. Las hojas y los tallos fueron separados y analizados para determinar el contenido de materia seca y proteína cruda. La producción de materia seca fue $23 \%$ superior y el contenido de proteína cruda fue significativamente mayor con el nitrógeno químico, mientras que el contenido de materia seca fue menor. No se encontraron diferencias significativas entre el tratamiento control y los tratamientos orgánicos.
\end{abstract}

Palabras clave: Morus alba, nitrógeno, fertilizante orgánico, producción orgánica, forrajes.

\begin{abstract}
Yield and quality of mulberry fodder (Morus alba) fertilized with with different fertilizers. A study was conducted at the "Alfredo Volio Mata" Experimental Station of the University of Costa Rica in order to evaluate the effect of applications of $150 \mathrm{~kg} \mathrm{~N} / \mathrm{ha}$ per $\mathrm{yr}$ from two organic fertilizers: vermicompost and compost; and from one chemical fertilizer, on mulberry fodder production and quality. The experimental period was 12 months, from July 2003 to July 2004. A 12-yr-old mulberry plantation planted at spacings of $0.9 \times 0.40 \mathrm{~m}$ (27777 plants/ha) was utilized in a randomized block design with four treatments: two organic fertilizers, ammonium nitrate $(33.5 \% \mathrm{~N})$, and a control. All plots were uniformly pruned at $0.6 \mathrm{~m}$ from the ground at the beginning of the trial. During the experimental period, plants were pruned consecutively every 90 days. Leaves and stems were separated and analyzed for dry matter and crude protein content. Dry matter production was $23 \%$ higher for the chemical fertilizer. Crude protein content was also significantly higher for the chemical nitrogen, while dry matter content was lower. The amount of $\mathrm{N}$ in the soil was sufficiently high for the control treatment to yield fodder and crude protein levels similar to those of organic fertilizers.
\end{abstract}

Keywords: Morus alba, nitrogen, organic fertilizer, organic production, forages.

\section{INTRODUCCIÓN}

La morera (Morus alba) es una especie forrajera que presenta características de calidad nutritiva, producción de biomasa y versatilidad agronómica importantes, representando así un excelente potencial para mejorar la calidad alimenticia de las dietas e incrementar la producción de los animales (Elizondo 2004).

\footnotetext{
Recibido: 11 de abril, 2007. Aceptado: 29 de agosto, 2007. Parte del proyecto inscrito en la Vicerrectoría de Investigación, No. 737-A4-049, Universidad de Costa Rica. Presentado en la Reunión Anual conjunta de la American Dairy Science Association, American Society of Animal Science, Asociación Mexicana de Producción Animal y Poultry Science Association. San Antonio, Texas. 8-12 de julio, 2007.

2 Estación Experimental Alfredo Volio Mata. Facultad de Ciencias Agroalimentarias. Universidad de Costa Rica, Cartago, Costa Rica. Correo electrónico: jaelizon@ cariari.ucr.ac.cr
} 
De acuerdo a Sánchez (2002), diversas investigaciones se han llevado a cabo en Japón, India, Tanzania, Kenya, Costa Rica, Colombia, Cuba, México, Guatemala y Brasil, que tienen que ver con la morera para alimentación animal. Dichos estudios han incluido aspectos agronómicos, modalidades de cosecha, formas de conservación y pruebas con animales que en general destacan el alto contenido de proteína en las hojas y los grandes rendimientos de biomasa por unidad de área, resaltando así la necesidad de reponer al suelo el nitrógeno extraído por las plantas en proporciones relativamente altas.

El nitrógeno $(\mathrm{N})$ es el elemento encontrado en mayores cantidades en el estiércol (Keeney y Hatfield 2001), el cuál durante muchos años ha sido utilizado como una forma para mejorar la fertilidad del suelo y la producción de los cultivos. En la actualidad, la industria química provee fertilizantes inorgánicos concentrados que son fácilmente distribuidos, utilizados y que pueden suplir la necesidad de este elemento por parte de los cultivos (Avnimelech 1986). Esto ha generado una disminución en el uso de fertilizantes orgánicos hasta un punto en el que la aplicación de fertilizantes inorgánicos, se ha llegado a convertir en un problema ambiental en muchos lugares del mundo (Butler et al. 2007).

Con el fin de utilizar tecnologías más amigables con el ambiente, y con el afán de reducir los costos de producción, gran número de productores están optando por la utilización de fertilizantes orgánicos. Entre los tipos de fertilizantes orgánicos más comúnmente utilizados se encuentran el compostaje y el lombriabono. El compostaje se refiere al abono orgánico obtenido mediante un proceso biológico aeróbico, en el cuál los microorganismos actúan sobre la materia rápidamente biodegradable (restos de cosecha, excrementos de animales y residuos urbanos). Por otra parte, el lombriabono se obtiene mediante la combinación de procesos, diseños y técnicas usadas sistemática e intensamente para cultivar grandes cantidades de ciertas especies de lombrices de tierra, para acelerar la estabilidad de desechos orgánicos, que son consumidos y digeridos por las lombrices con la ayuda de microorganismos aeróbicos y anaeróbicos; y convertidos a un material fecal que es mucho más fino, con mayor contenido de humus y microbialmente más activo que el material original, donde los nutrientes se encuentran en una forma más soluble y disponible para las plantas (Aranda et al. 1999).

A pesar de las ventajas que pueden presentar ambos tipos de abonos orgánicos, es poco lo que se conoce sobre su utilización en cultivos de alto rendimiento forrajero como la morera y no existe literatura que haya comparado los rendimientos de este cultivo cuando se utilizan fertilizantes orgánicos o fertilizantes químicos. Por esta razón, el presente trabajo tuvo como objetivo evaluar la aplicación de $150 \mathrm{~kg}$ de nitrógeno por hectárea por año, proveniente de dos fertilizantes orgánicos y un fertilizante químico sobre la producción de biomasa y proteína cruda de la morera.

\section{MATERIALES Y MÉTODOS}

El trabajo de campo se llevó a cabo en la Estación Experimental "Alfredo Volio Mata" de la Universidad de Costa Rica, ubicada en la provincia de Cartago a $1.542 \mathrm{msnm}$, con una precipitación media anual de $2.050 \mathrm{~mm}$, distribuida durante los meses de mayo a noviembre. La humedad relativa media es de $84 \%$ y la temperatura media de $19,5^{\circ} \mathrm{C}$. El suelo es de origen volcánico, clasificado como Typic Distrandepts (Vásquez 1982), caracterizado por tener una profundidad media con buen drenaje natural y fertilidad media (Cuadro 1). El experimento se inició en julio del 2003 y finalizó en julio del 2004.

Ecológicamente la zona se tipifica como Bosque Húmedo Montano Bajo (Tosi 1970 citado por Vásquez 1982).

Se utilizó una plantación de morera con 12 años de establecida en un área de 1.000 metros cuadrados con una densidad de siembra de 27.777 plantas/ha $(0,40 \mathrm{~m}$ entre plantas y 0,90 m entre hileras). El área total se dividió en tres grandes bloques. Cada bloque fue subdividido en cuatro parcelas para los diferentes tratamientos de fertilización nitrogenada a un nivel constante de 150 $\mathrm{kg} / \mathrm{ha} / \mathrm{año}$, la cuál se dividió en dos aplicaciones iguales durante la época de lluvia. Se utilizó dicho nivel de nitrógeno, ya que es la dosis utilizada por los productores. Los tratamientos utilizados fueron lombriabono (23 t/ha/año), compostaje (22 t/ha/año), nitrato de amonio $(33,5 \% \mathrm{~N})$ y un tratamiento testigo (0 nitrógeno), con su 
Cuadro 1. Análisis químico inicial del suelo sembrado con el cultivo de morera. Cartago, Costa Rica. 2004.

\begin{tabular}{|c|c|c|c|c|c|c|c|c|c|c|c|c|}
\hline \multirow[b]{2}{*}{ pH } & \multirow[b]{2}{*}{ CICE } & \multicolumn{4}{|c|}{$\operatorname{cmol}(+) / l$} & \multicolumn{5}{|c|}{$\mathrm{mg} / \mathrm{l}$} & \multicolumn{2}{|c|}{$\%$} \\
\hline & & $\mathbf{C a}$ & Mg & $\mathbf{K}$ & Acidez & $\mathbf{P}$ & $\mathrm{Cu}$ & $\mathbf{F e}$ & Mn & Zn & NTotal & MO \\
\hline 5,6 & 11,8 & 8,4 & 1,8 & 1,4 & 0,2 & 11,9 & 14,6 & 128,0 & 13,0 & 2,7 & 0,3 & 6,0 \\
\hline
\end{tabular}

composición presentada en el Cuadro 2. El lombriabono fue producido a partir de estiércol bovino y el compostaje se produjo a partir de residuos de jardín (hojas, zacate, arbustos). Una muestra representativa de cada tipo de abono fue enviada al Centro de Investigaciones Agronómicas de la Universidad de Costa Rica para su respectivo análisis químico.

Cuadro 2. Análisis químico de los abonos orgánicos utilizados en el experimento. Cartago, Costa Rica. 2004.

\begin{tabular}{lccccccc}
\hline & & \multicolumn{3}{c}{$\mathbf{c m o l}(+) / \mathbf{l}$} & & \multicolumn{3}{c}{$\%$} \\
\cline { 3 - 4 } \cline { 7 - 8 } Abono & pH & $\mathbf{C a}$ & $\mathbf{M g}$ & $\mathbf{K}$ & & $\mathbf{N}$ Total & $\mathbf{H}_{2} \mathbf{O}$ \\
\hline Lombriabono & 7,20 & 1,90 & 0,67 & 1,34 & & 1,64 & 60,11 \\
Compost & 7,05 & 1,72 & 0,45 & 0,63 & & 1,10 & 37,14 \\
\hline
\end{tabular}

$\mathrm{Al}$ inicio del experimento, las plantas de morera se podaron de manera uniforme a $60 \mathrm{~cm}$ de altura sobre el nivel del suelo. A partir de esta uniformización se programaron cuatro cortes consecutivos cada 90 días.

Se empleó una estructura experimental de bloques completos al azar (Kuehl 2000), utilizando el siguiente modelo matemático:

$$
\mathrm{Y}_{\mathrm{ijk}}=\mu+\alpha_{\mathrm{i}}+\beta_{\mathrm{j}}+\gamma_{\mathrm{k}}+\varepsilon_{\mathrm{ijk}}
$$

Donde:

$\mathrm{Y}_{\mathrm{ij \textrm {k }}}=$ Producción en $\mathrm{kg} / \mathrm{ha} / \mathrm{año}$

$\mu=$ Media general

$\alpha_{i}=$ Efecto del i-ésimo tratamiento

$\beta_{\mathrm{j}}=$ Efecto del j-ésimo bloque

$\gamma_{\mathrm{k}}=$ Efecto del k-ésimo muestreo

$\varepsilon_{\mathrm{ijk}}=$ Error experimental

Al cabo de cada periodo experimental de rebrote, las parcelas fueron cosechadas totalmente a la misma altura del corte de uniformización. La producción de biomasa fresca se pesó en el campo y se extrajo una muestra aleatoria del $10 \%$ de plantas enteras de cada parcela y se separaron en tallos y hojas. Cada componente fue pesado en fresco y secado a $60^{\circ} \mathrm{C}$ durante 48 horas. Las muestras se molieron en un molino Willey, con una malla de un milímetro. Posteriormente se determinó el contenido de materia seca y proteína cruda siguiendo los métodos aprobados por el AOAC (2002). Se estimaron los rendimientos de biomasa verde, biomasa seca y proteína cruda por hectárea en la planta entera, hojas y tallos a partir de los muestreos realizados.

Los datos obtenidos se analizaron con el PROC GLM del paquete estadístico SAS (1999) de acuerdo al modelo propuesto. Las fuentes que resultaron estadísticamente diferentes $(\mathrm{P}<0,05)$ se sometieron a la prueba de Waller-Duncan.

\section{RESULTADOS Y DISCUSIÓN}

El rendimiento anual de materia verde, materia seca y proteína cruda de la planta entera de morera, hojas y tallos, obtenido de los cortes secuenciales efectuados a través del experimento para cada uno de los tratamientos se encuentra en el Cuadro 3.

Se encontraron diferencias altamente significativas $(\mathrm{P}<0,01)$ para las diferentes variables de producción evaluadas. El rendimiento total de materia verde fue de 93,7; 94,2; 123,7 y 93,4 t/ha/año para el lombriabono, compostaje, nitrato de amonio y tratamiento control, respectivamente. Estos rendimientos se encuentran dentro del rango observado por Boschini et al. (1998) y Espinoza (1996) quienes en explotaciones intensivas localizadas en zonas de alta luminosidad, con suelos bien drenados y con precipitaciones media o alta obtuvieron rendimientos de biomasa fresca de 70-119 t/ha/año. 
Cuadro 3. Producción anual y composición química de las hojas, tallos y planta entera de morera fertilizada con tres tipos de abono a razón de $150 \mathrm{~kg}$ de N/ha/año, Cartago, Costa Rica, 2004.

\begin{tabular}{lcccccc}
\hline & \multicolumn{5}{c}{ Tratamiento } \\
\cline { 2 - 7 } Variable & Lombriabono & Compostaje & Químico & Control1 & SEM & P \\
\hline \multicolumn{2}{l}{ Materia verde, kg/ha/año } & & & & & \\
Hoja & $55.283 \mathrm{~b}$ & $56.940 \mathrm{~b}$ & $70.174 \mathrm{a}$ & $55.259 \mathrm{~b}$ & 2.557 & 0,001 \\
Tallo & $38.447 \mathrm{~b}$ & $37.317 \mathrm{~b}$ & $53.573 \mathrm{a}$ & $38.646 \mathrm{~b}$ & 2.440 & 0,001 \\
Total & $93.729 \mathrm{~b}$ & $94.257 \mathrm{~b}$ & $123.747 \mathrm{a}$ & $93.905 \mathrm{~b}$ & 4.599 & 0,001
\end{tabular}

Materia seca, \%

$\begin{array}{ccccccc}\text { Hoja } & 22,7 \mathrm{a} & 22,6 \mathrm{a} & 21,5 \mathrm{~b} & 22,7 \mathrm{a} & 0,3 & 0,001 \\ \text { Tallo } & 23,3 & 23,1 & 22,2 & 23,5 & 0,5 & \text { NS } \\ \text { Total } & 22,8 \mathrm{a} & 22,8 \mathrm{a} & 21,7 \mathrm{~b} & 22,9 \mathrm{a} & 0,3 & 0,001\end{array}$

\section{Materia seca, $\mathrm{kg} / \mathrm{ha} / \mathbf{a n ̃ o}$}

$\begin{array}{lrrrrrr}\text { Hoja } & 12.604 \mathrm{~b} & 12.943 \mathrm{~b} & 15.153 \mathrm{a} & 12.601 \mathrm{~b} & 546 & 0,001 \\ \text { Tallo } & 8.807 \mathrm{~b} & 8.638 \mathrm{~b} & 11.413 \mathrm{a} & 8.875 \mathrm{~b} & 602 & 0,001 \\ \text { Total } & 21.411 \mathrm{~b} & 21.581 \mathrm{~b} & 26.567 \mathrm{a} & 21.476 \mathrm{~b} & 1.039 & 0,001\end{array}$

Proteína cruda, \%

$\begin{array}{lcccccc}\text { Hoja } & 15,7 \mathrm{~b} & 15,3 \mathrm{~b} & 17,3 \mathrm{a} & 16,0 \mathrm{~b} & 0,3 & 0,001 \\ \text { Tallo } & 5,7 & 5,3 & 6,3 & 5,5 & 0,3 & \text { NS } \\ \text { Total } & 11,7 \mathrm{~b} & 11,5 \mathrm{~b} & 12,6 \mathrm{a} & 11,7 \mathrm{~b} & 0,3 & 0,001\end{array}$

Proteína cruda, kg/ha/año

\begin{tabular}{lrrrrrr} 
Hoja & $1982 \mathrm{~b}$ & $2012,8 \mathrm{~b}$ & $2663,2^{\mathrm{a}}$ & $2025,6 \mathrm{~b}$ & 106,8 & 0,001 \\
Tallo & $514 \mathrm{~b}$ & $464,4 \mathrm{~b}$ & $773,2^{\mathrm{a}}$ & $488,4 \mathrm{~b}$ & 48,4 & 0,001 \\
Total & $2496 \mathrm{~b}$ & $2476,8 \mathrm{~b}$ & $3437,2^{\mathrm{a}}$ & $2514,4 \mathrm{~b}$ & 145,2 & 0,001 \\
\hline
\end{tabular}

a,b,c representan diferencias significativas en una misma fila.

${ }^{1}$ Sin fertilización.

El rendimiento total de materia seca para cada uno de los tratamientos se puede observar en la Figura 1. El tratamiento químico presentó la mayor respuesta con 26,6 t/ ha/año, mientras que con los tratamientos restantes se obtuvo un promedio de 21,5 t/ha/año, lo cuál indica que con el fertilizante químico se obtuvo una producción anual de materia seca superior al 20\% con respecto a los demás tratamientos. Sin embargo, este rendimiento es muy similar a los reportados en otros ensayos. En Guatemala, Rodríguez et al. (1992) han informado de rendimientos de 2 a $26 \mathrm{t} / \mathrm{ha} / \mathrm{año}$ de materia seca con plantas enteras, cosechadas a $30 \mathrm{~cm}$ sobre el nivel del suelo, en intervalos de poda de 6 a 12 semanas y con niveles de fertilización de 0 a $80 \mathrm{~kg}$ de N/ha/año. En otro estudio, Benavides et al. (1994) emplearon niveles de fertilización nitrogenada de 0 a 


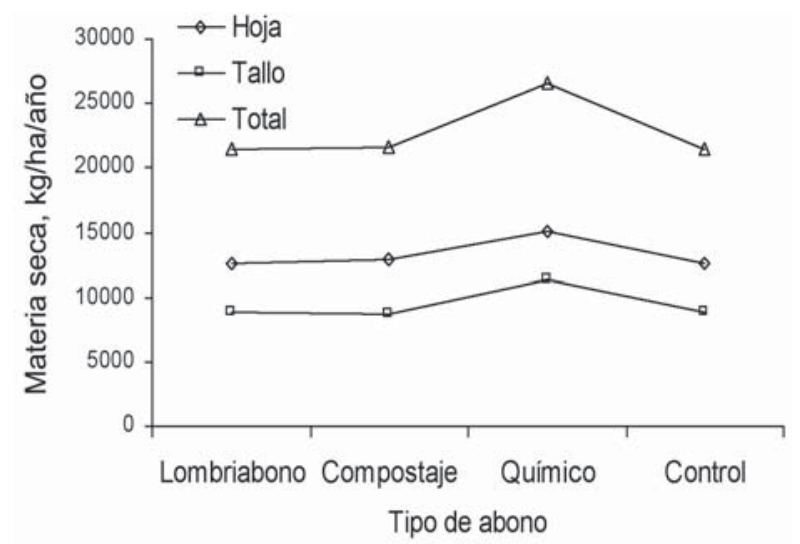

Figura 1. Producción de materia seca de las hojas, tallos y planta enterada de morera fertilizada con diferentes tipos de abonos a razón de $150 \mathrm{~kg}$ de N/ha/año. Cartago, Costa Rica. 2004.

$480 \mathrm{~kg} / \mathrm{ha} / \mathrm{año}$ durante tres años, logrando rendimientos de materia seca de 19 a 30 t/ha/año. Por su parte, Boschini et al. (1999) al utilizar una dosis de $150 \mathrm{~kg}$ de nitrógeno/ha/año, con diferentes distancias de siembra $(0,60$ a $1,20 \mathrm{~m})$ y diferentes frecuencias de corte $(56$ a 112 días) determinaron que la producción anual de materia seca promedio fue de 19,7; 9,9 y 9,8 t/ha/año para la planta entera, tallos y hojas, respectivamente.

La producción de proteína cruda por hectárea y por año tanto en la planta entera como en los tallos $\mathrm{y}$ hojas fue significativamente superior $(\mathrm{P}<0,01)$ con

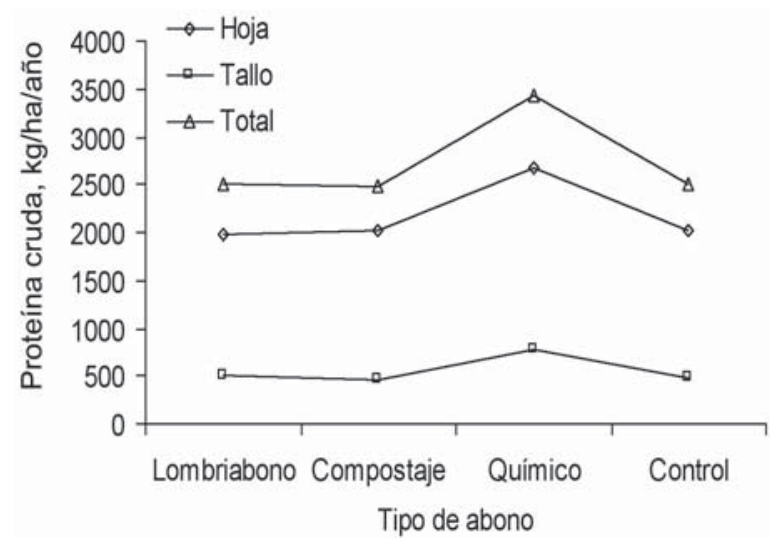

Figura 2. Producción de proteína cruda de las hojas, tallos y planta entera de morera fertilizada con diferentes tipos de abono, a razón de $150 \mathrm{~kg}$ de N/ha/año. Cartago, Costa Rica. 2004. el tratamiento químico que con los otros tratamientos utilizados. En la Figura 2 puede observarse como con el tratamiento químico se obtuvieron los mayores rendimientos de proteína cruda, mientras que no se observaron diferencias significativas entre los fertilizantes orgánicos y el tratamiento control. Es interesante observar que con el tratamiento químico se obtuvo cerca de 941 kg más de proteína cruda sobre el promedio de los demás tratamientos.

El contenido de materia seca y proteína cruda de la planta entera de morera, hojas y tallos, obtenido de los cortes secuenciales efectuados a través del experimento para cada uno de los tratamientos utilizados se presenta en el Cuadro 2. Se encontraron diferencias altamente significativas $(\mathrm{P}<0,01)$ para las diferentes variables nutricionales evaluadas con respecto a los tratamientos utilizados. El contenido de materia seca de la planta entera fue de $21,67 \%$ para el tratamiento químico, mientras que con el resto de los tratamientos se obtuvo en promedio un valor de 22,83\%. Estos valores son superiores a los encontrados por Boschini (2001) que reporta un promedio de $17,32 \%$. Por su parte, el contenido de proteína cruda en la planta entera fue significativamente mayor $(\mathrm{P}<0,01)$ para el tratamiento químico con un valor de $12,58 \%$, mientras que los demás tratamientos presentaron un valor promedio de $11,6 \%$. Los valores encontrados son similares a los obtenidos por Rodríguez et al. (1992) quienes reportaron un valor de 10,97 $( \pm 3,53)$. Sin embargo, Boschini (2001) reporta valores superiores con un promedio de $16,5 \%$.

En general, puede observarse que con el tratamiento químico se obtuvieron los mayores rendimientos de biomasa y proteína cruda. Es conocido que la aplicación de $\mathrm{N}$ químico resulta en el corto plazo, en rendimientos superiores a los obtenidos con abonos orgánicos, ya que el $\mathrm{N}$ inorgánico presente en el fertilizante químico está fácilmente disponible para ser utilizado por las plantas, mientras que el $\mathrm{N}$ orgánico debe ser mineralizado para poder ser asimilado por las plantas, tal como se explicará más adelante. Es por esta razón que el uso de abonos orgánicos presenta un gran reto para sincronizar la disponibilidad de nitrógeno con la demanda de las plantas, cuando se compara con el uso de fertilizantes sintéticos y además, el $\mathrm{N}$ que no se utiliza, puede ser mineralizado fuera del ciclo productivo, presentando el inconveniente de 
estar propenso a perderse por lixiviación (Korsaeth et al. 2003). Por su parte, no se observaron diferencias significativas entre los tratamientos donde se utilizó abono orgánico con respecto al tratamiento testigo. El nitrógeno contenido en los abonos orgánicos y aquel presente en el suelo se encuentran en forma orgánica e inorgánica, con $95 \%$ o más presente en forma orgánica (White 2006). El nitrógeno inorgánico es obtenido durante la descomposición microbiana de la materia orgánica en el proceso de amonificación, de acuerdo a la siguiente reacción:

Nitrógeno orgánico (proteínas, ácidos nucleicos) $\rightarrow \mathrm{NH}_{4}^{+}+\mathrm{OH}^{-}$

Sujeto a cualquier limitación impuesta por la temperatura, humedad y aireación del suelo, el $\mathrm{NH}_{4}^{+}$es oxidado a $\mathrm{NO}_{3}^{-}$en el proceso de nitrificación, representado por la siguiente reacción:

$$
\mathrm{NH}_{4}^{+}+2 \mathrm{O}_{2} \rightarrow \mathrm{NO}_{3}^{-}+\mathrm{H}_{2} \mathrm{O}+2 \mathrm{H}^{+}
$$

Las dos reacciones anteriores describen el proceso conocido como mineralización (White 2006). Dicho proceso se acelera con un incremento en la temperatura y aumenta con una adecuada humedad y una buena disponibilidad de oxígeno (Jarvis et al. 1995). Una vez que el $\mathrm{N}$ orgánico ha sido mineralizado, las plantas lo puedan utilizar. La cantidad de $\mathrm{N}$ orgánico que se puede mineralizar, es el $\mathrm{N}$ orgánico multiplicado por un factor de mineralización que puede oscilar entre 0,25 y 0,35 (Van y Reeves 2000). Debido a lo explicado anteriormente, la falta de respuesta de la morera a la fertilización orgánica en este experimento puede atribuirse a un bajo nivel de mineralización del nitrógeno orgánico, lo que limita la cantidad de nitrógeno inorgánico disponible para las plantas al nitrógeno presente en el suelo, el cuál es prácticamente el mismo nivel disponible para las plantas del tratamiento control. Es importante recalcar que la remoción de nitrógeno en el tratamiento control fue de poco más de $400 \mathrm{~kg} / \mathrm{ha} / \mathrm{año}$. La continua remoción de esta cantidad de nitrógeno agotará las reservas de cualquier suelo, por lo que es importante evaluar la producción del tratamiento testigo a lo largo de varios años con respecto a los tratamientos orgánicos.

\section{CONCLUSIONES}

Los mayores rendimientos de materia verde, materia seca y proteína cruda en $\mathrm{kg} / \mathrm{ha} / \mathrm{año}$ se obtuvieron con la fertilización química. El contenido de materia seca (\%) en la planta entera fue menor con el tratamiento químico, sin embargo, el contenido de proteína cruda fue mayor. No se encontraron diferencias significativas entre los diferentes abonos orgánicos y el tratamiento control, lo cuál se atribuye a bajas tasas de mineralización del nitrógeno en los abonos orgánicos.

\section{LITERATURA CITADA}

A.O.A.C. (Association of Official Analysis Chemistry). 2002. Methods of Analysis. 13 ed. Washington D.C. U.S.A.

ARANDA, E.; BAROIS, I.; ARELLANO, P.; IRISSON, S.; SALAZAR, T.; RODRIGUEZ, J.; PATRON, J. 1999. In: Lavelle, P.; Brussaard, L.; Hendrix, P. eds. Earthworm management in tropical agroecosystems. CABI Publishing. London, UK. CABI Publishing.

AVNIMELECH, Y. 1986. Organic residues in modern agriculture. In: Chen, Y.; Avnimelech, Y. eds. The role of organic matter in modern agriculture. The Netherlands. Martinus Nijhoff Publishers.

BENAVIDES, J.; LACHAUX, M.; FUENTES, M. 1994. Efecto de la aplicación de estiércol de cabra en el suelo sobre la calidad y producción de biomasa de Morera (Morus sp.). Árboles y arbustos forrajeros en América Central. Serie Técnica. San José, Costa Rica. CATIE. Informe Técnico No 236. Volumen II.

BOSCHINI, C. 2001. Producción y calidad de la morera (Morus alba) cosechada en diferentes modalidades de poda. Agronomía Mesoamericana 12(2):175-180.

BOSCHINI, C., DORMOND, H., CASTRO, A. 1998. Producción de biomasa de la morera (Morus alba) en la Meseta Central de Costa Rica, establecida y cosechada a diferentes distancias de siembra, alturas y frecuencias de poda. Agronomía Mesoamericana 9(2):28-39. 
BOSCHINI, C.; DORMOND, H.; CASTRO, A. 1999. Respuesta de la morera (Morus alba) a la fertilización nitrogenada, densidades de siembra y a la defoliación. Agronomía Mesoamericana 10(2):07-16.

BUTLER, D.M.; RANELLS, N.N.; FRANKLIN, D.H.; POORE, M.H.; GREEN, J.T. 2007. Ground cover impacts on nitrogen export from manured riparian pasture. J. Environ. Qual. 36:155-162.

ELIZONDO, J. 2004. Calidad nutricional y consumo de morera (Morus alba), Ramio (Bohemeria nivea (L) GAUD) y sorgo negro forrajero (Sorghum almum) en cabras. Agronomía Mesoamericana 15(2):209-213.

ESPINOZA, E. 1996. Efecto del sitio y del nivel de fertilización nitrogenada sobre la producción y calidad de tres variedades de morera en Costa Rica. Tesis de Maestría. Turrialba, Costa Rica. CATIE.

JARVIS, S.; SCHOLEFIELD, D.; PAIN, B. 1995. Nitrogen cycling in grazing systems. In: Bacon, P. ed. Nitrogen fertilization in the environment. Marcel Dekker, Inc. N.Y, USA. Marcel Dekker, Inc.

KEENEY, D.; HATFIELD, J. 2001. The nitrogen cycle, historical perspective and current, and potential future concerns. In: Follett, R.; Hatfield, J. eds. Nitrogen in the environment: Sources, problems and management. The Netherlands. Elsevier Science.

KORSAETH, A.; BAKKEN, L.R; HUGH RILEY, H. 2003. Nitrogen dynamics of grass as affected by $\mathrm{N}$ input regimes, soil texture and climate: lysimeter measurements and simulations. Nutrient Cycling in Agroecosystems 66:181-199, 2003.

KUEHL, R.O. 2000. Design of experiments: statistical principles of research design and analysis. 2 ed. California, U.S.A. Duxbury Press.

RODRIGUEZ, C.; QUIÑONEZ, J.; ARIAS, R. 1992. Frecuencias de corte y niveles de fertilización nitrogenada en rendimiento y calidad del forraje de morera (Morus sp.), en Cuyuta, Guatemala. Agronomía Mesoamericana 3:48-51.

SÁNCHEZ, M. 2002. World distribution and utilization of mulberry and its potential for animal feeding. In: Sánchez, M. ed. Proceedings of an electronic conference. FAO. Roma.

SAS. 1999. Statistical analysis system. SAS User's Guide; Statistics (Versión 8 Ed.). Cary, NC. SAS Institute Inc.

VAN, J.; REEVES, J. 2000. On farm quick tests for estimating nitrogen in dairy manure. Journal of Dairy Science (83): 1837-1844.

VASQUEZ, A. 1982. Estudio detallado de los suelos de la Estación Experimental de Ganado Lechero El Alto. Tesis de Licenciatura. San José, Costa Rica. Escuela de Fitotecnia, Facultad de Agronomía, Universidad de Costa Rica.

WHITE, R.E. 2006. Principles and practice of soil science: The soil as a natural resource. 4 ed. Great Britain. Blackwell Publishing. 